\title{
Essential Self-Adjointness of Anticommutative Operators
}

\author{
Toshimitsu Takaesu \\ Faculty of Science and Technology, Gunma University, Gunma 376-8515, Japan \\ Correspondence should be addressed to Toshimitsu Takaesu; t-takaesu@hotmail.co.jp \\ Received 30 January 2014; Accepted 6 March 2014; Published 27 March 2014 \\ Academic Editor: Jen-Chih Yao
}

Copyright (C) 2014 Toshimitsu Takaesu. This is an open access article distributed under the Creative Commons Attribution License, which permits unrestricted use, distribution, and reproduction in any medium, provided the original work is properly cited.

The self-adjoint extensions of symmetric operators satisfying anticommutation relations are considered. It is proven that an anticommutative type of the Glimm-Jaffe-Nelson commutator theorem follows. Its application to an abstract Dirac operator is also considered.

\section{Introduction and Main Theorem}

In this paper, we consider the essential self-adjointness of anticommutative symmetric operators. Let $H$ be a symmetric operator on a Hilbert space $\mathscr{H}$; that is, $H$ satisfies $H \quad C$ $H^{*}$. It is said that $H$ is self-adjoint if $H=H^{*}$ and $H$ is essentially self-adjoint if its closure $\bar{H}$ is self-adjoint. We are interested in conditions under which a symmetric operator is essentially self-adjoint. The Glimm-Jaffe-Nelson commutator theorem (e.g., [1, Theorem 2.32], [2, Theorem X.36]) is one criterion for the essential self-adjointness of commutative symmetric operators. The commutator theorem shows that if a symmetric operator $H$ and a self-adjoint operator $X$ obey a commutation relation on a dense subspace $\mathscr{D}$, which is a core of $X$, then $H$ is essentially self-adjoint on $\mathscr{D}$. Historically, Glimm and Jaffe [3] and Nelson [4] investigate the commutator theorem for quantum field models. Faris and Lavine [5] apply it to quantum mechanical models and Fröhlich [6] considers a generalization of the commutator theorem and proves that a multiple commutator formula follows. Here, we overview the commutator theorem.

Let $H$ and $X$ be linear operators on $\mathscr{H}$. Assume the following conditions.

(C.1) $H$ is symmetric and $X$ is self-adjoint.

(C.2) There exists $\delta_{X}>0$ such that, for all $\Psi \in \mathscr{D}(X)$,

$$
\delta_{X}(\Psi, \Psi) \leq|(\Psi, X \Psi)|
$$

(C.3) $X$ has a core $\mathscr{D}_{0}$ satisfying $\mathscr{D}_{0} \subset \mathscr{D}(H)$, and there exist constants $a \geq 0$ and $b \geq 0$ such that, for all $\Psi \in$ $\mathscr{D}_{0}$,

$$
\|H \Psi\| \leq a\|X \Psi\|+b\|\Psi\|
$$

Theorem A (Glimm-Jaffe-Nelson commutator theorem). Let $H$ and $X$ be operators satisfying (C.1)-(C.3). Suppose (i) or (ii) as follows.

(i) There exists a constant $c_{1} \geq 0$ such that, for all $\Psi \in \mathscr{D}_{0}$,

$$
|(H \Psi, X \Psi)-(X \Psi, H \Psi)| \leq c_{1}|(\Psi, X \Psi)| .
$$

(ii) There exists a constant $c_{2} \geq 0$ such that, for all $\Psi \in \mathscr{D}_{0}$,

$$
c_{2}|(\Psi, X \Psi)| \leq|(H \Psi, X \Psi)-(X \Psi, H \Psi)| .
$$

Then, $H$ is essentially self-adjoint on $\mathscr{D}_{0}$.

Remark 1. In the commutator theorem, condition (i) is usually supposed. It is also proven under condition (ii) in a similar way to Theorem 2 .

The idea of the proof of the commutator theorem is as follows. Let $X$ and $Y$ be symmetric operators on a Hilbert space. Then, the real part and the imaginary part of the inner product $(X \Psi, Y \Psi)$ for $\Psi \in \mathscr{D}(X Y) \cap \mathscr{D}(Y X)$ are expressed by

$$
\begin{aligned}
& \operatorname{Re}(X \Psi, Y \Psi)=\frac{1}{2}(\Psi,\{X, Y\} \Psi), \\
& \operatorname{Im}(X \Psi, Y \Psi)=\frac{1}{2 i}(\Psi,[X, Y] \Psi),
\end{aligned}
$$


respectively, where $\{X, Y\}=X Y+Y X$ and $[X, Y]=X Y-Y X$. In the proof of the commutator theorem, the imaginary part is estimated. In Theorem 2, we prove that an anticommutative symmetric operator is essentially self-adjoint on a dense subspace by estimating the real part.

Theorem 2. Assume (C.1)-(C.3). In addition, suppose that (I) or (II) holds.

(I) There exists a constant $d_{1} \geq 0$ such that, for all $\Psi \in \mathscr{D}_{0}$,

$$
|(H \Psi, X \Psi)+(X \Psi, H \Psi)| \leq d_{1}|(\Psi, X \Psi)| .
$$

(II) There exists a constant $d_{2} \geq 0$ such that, for all $\Psi \in \mathscr{D}_{0}$,

$$
d_{2}|(\Psi, X \Psi)| \leq|(H \Psi, X \Psi)+(X \Psi, H \Psi)|
$$

Then, $H$ is essentially self-adjoint on $\mathscr{D}_{0}$.

Proof of Theorem 2. We show that, for some $z \in \mathbf{C} \backslash \mathbf{R}$, $\operatorname{dim} \operatorname{ker}\left(\left(H_{\left\lceil D_{0}\right.}\right)^{*}+z^{\sharp}\right)=0$ where $z^{\sharp}=z, z^{*}$. Let $\Psi \in$ $\mathscr{D}\left(\left(H_{\left\lceil\mathscr{D}_{0}\right.}\right)^{*}\right)$ and let $\Xi=X^{-1} \Psi$. Since $\left(\left(H_{\left\lceil\mathscr{D}_{0}\right.}\right)^{*}\right)^{*}=\overline{H_{\left\lceil\mathscr{D}_{0}\right.}}$, we have

$$
\begin{aligned}
\operatorname{Re}(\Xi & \left.,\left(\left(H_{\left\lceil\mathscr{D}_{0}\right.}\right)^{*}+z^{\sharp}\right) \Psi\right) \\
= & \frac{1}{2}\left(\left(\overline{H_{\left\lceil\mathscr{D}_{0}\right.}} \Xi, X \Xi\right)+\left(X \Xi, \overline{H_{\left\lceil\mathscr{D}_{0}\right.}} \Xi\right)\right) \\
& +\operatorname{Re} z(\Xi, X \Xi) .
\end{aligned}
$$

First, we assume that (I) holds. Let $z \in \mathbf{C} \backslash \mathbf{R}$ satisfy $|\operatorname{Re} z|>$ $d_{1} / 2$. Since $\mathscr{D}_{0}$ is a core of $X$, it follows from (C.3) and (I) that $\mathscr{D}(X) \subset \mathscr{D}\left(\overline{H_{\left\lceil\mathscr{D}_{0}\right.}}\right)$ and for all $\Phi \in \mathscr{D}(X)$,

$$
\left|\left(\overline{H_{\left\lceil\mathscr{D}_{0}\right.}} \Phi, X \Phi\right)+\left(X \Phi, \overline{H_{\left\lceil\mathscr{D}_{0}\right.}} \Phi\right)\right| \leq d_{1}|(\Phi, X \Phi)| .
$$

By (8) and (9), we have

$$
\begin{aligned}
\left|\operatorname{Re}\left(\Xi,\left(\left(H_{\left\lceil\mathscr{D}_{0}\right.}\right)^{*}+z^{\sharp}\right) \Psi\right)\right| & \geq\left(|\operatorname{Re} z|-\frac{d_{1}}{2}\right)|\Xi, X \Xi| \\
& \geq \delta_{X}\left(|\operatorname{Re} z|-\frac{d_{1}}{2}\right)(\Xi, \Xi) .
\end{aligned}
$$

Since $\Psi \in \operatorname{ker}\left(\left(H_{\left\lceil\mathscr{D}_{0}\right.}\right)^{*}+z^{\sharp}\right)$, we have $\Xi=X^{-1} \Psi=0$ from (10). Then, we have $\Psi=0$. Next, we suppose that (II) follows. Let $z \in \mathbf{C} \backslash \mathbf{R}$ satisfy $|\operatorname{Re} z|<d_{2} / 2$. Since $\mathscr{D}_{0}$ is a core of $X$, it also follows from (C.3) and (II) that $\mathscr{D}(X) \subset \mathscr{D}\left(\overline{H_{\left\lceil D_{0}\right.}}\right)$ and for all $\Phi \in \mathscr{D}(X)$,

$$
d_{2}|(\Phi, X \Phi)| \leq\left|\left(\overline{H_{\left\lceil\mathscr{D}_{0}\right.}} \Phi, X \Phi\right)+\left(X \Psi, \overline{H_{\left\lceil\mathscr{D}_{0}\right.}} \Phi\right)\right| .
$$

Then from (8) and (11), we have

$$
\begin{aligned}
\left|\operatorname{Re}\left(\Xi,\left(\left(H_{\mid \mathscr{D}_{0}}\right)^{*}+z^{\sharp}\right) \Psi\right)\right| & \geq\left(\frac{d_{2}}{2}-|\operatorname{Re} z|\right)|\Xi, X \Xi| \\
& \geq \delta_{X}\left(\frac{d_{2}}{2}-|\operatorname{Re} z|\right)(\Xi, \Xi) .
\end{aligned}
$$

Since $\Psi \in \operatorname{ker}\left(\left(H_{\mid \mathscr{D}_{0}}\right)^{*}+z^{\sharp}\right)$ and $\Xi=X^{-1} \Psi$, we have $\Psi=0$ from (12). Thus, the proof is obtained.

\section{Application of Theorem 2}

We apply Theorem 2 to abstract Dirac operator theory [1, 7]. Let $\mathscr{H}$ be a Hilbert space. Let $H$ and $\tau$ be self-adjoint operators on $\mathscr{H}$. Assume that $\tau$ is bounded, $\tau^{2}=I$ and, $\tau \mathscr{D}(H) \subset \mathscr{D}(H)$. Then $H$ is called an abstract Dirac operator on $\mathscr{H}$ with unitary involution $\tau$. We construct an abstract Dirac operator by weakly commuting operators. Let $X$ and $Y$ be densely defined linear operators on a Hilbert space. The weak commutator of $X$ and $Y$ is defined for $\Phi \in \cap \mathscr{D}\left(X^{*}\right) \cap$ $\mathscr{D}\left(Y^{*}\right)$ and for $\Psi \in \mathscr{D}(X) \cap \mathscr{D}(Y)$ by

$$
[X, Y]^{0}(\Phi, \Psi)=\left(X^{*} \Phi, Y \Psi\right)=\left(Y^{*} \Phi, X \Psi\right) .
$$

Let $\left\{P_{j}\right\}_{j=1}^{N}, N \in \mathbf{N}$, be self-adjoint operators on a Hilbert space $\mathscr{H}$. Set $\mathscr{D}_{0}=\cap_{j=1}^{N} \mathscr{D}\left(P_{j}\right)$. Assume that $\left\{P_{j}\right\}_{j=1}^{N}$ satisfy the following condition.

(S.1) $\mathscr{D}_{0}$ is dense in $\mathscr{H}$. For all $\Phi, \Psi \in \mathscr{D}_{0},\left[P_{j}, P_{l}\right]^{0}(\Phi, \Psi)=$ $0, j, l,=1, \ldots, N$.

Let $M$ be a bounded self-adjoint operator $\mathscr{H}$ satisfying the following condition.

(S.2) For all $\Phi, \Psi \in \mathscr{D}_{0},\left[M, P_{j}\right]^{0}(\Phi, \Psi)=0, j=1, \ldots, N$.

Let $\mathscr{K}$ be a Hilbert space. Let $\left\{\Gamma_{j}\right\}_{j=1}^{N}$ and $B$ be bounded self-adjoint operators on $\mathscr{K}$ satisfying the following anticommutation relations:

(S.3) (i) $\left\{\Gamma_{j}, \Gamma_{k}\right\}=2 \delta_{j, k}, j, k=1, \ldots, N$, (ii) $\left\{\Gamma_{j}, B\right\}=$ $1, j=1, \ldots, N$, (iii) $B^{2}=I$.

Then, the next assertion holds.

Theorem 3. Let $\mathscr{H}_{D}=\mathscr{K} \otimes \mathscr{H}$. Assume (S.1)-(S.3). Then,

$$
H_{D}=\sum_{j=1}^{N} \Gamma_{j} \otimes P_{j}+B \otimes M
$$

is self-adjoint on $\mathscr{D}\left(H_{D}\right)=\mathscr{K} \otimes \mathscr{D}_{0}$.

Remark 4. In the case where $\left\{P_{j}\right\}_{j=1}^{N}$ strongly commute, Theorem 3 has been proven ([8, Theorem 4.3], [9, Lemma $6.7])$ by strongly anticommuting methods $[10,11]$.

It is seen that $(I \otimes B)^{2}=I$ and $(I \otimes B) \mathscr{D}\left(H_{D}\right) \subset \mathscr{D}\left(H_{D}\right)$. Then, from Theorem $3, H_{D}$ is an abstract Dirac operator on $\mathscr{H}_{D}$ with the unitary involution $I \otimes B$.

To prove Theorem 3, we show some lemmas.

Lemma 5. Let $\left\{C_{j}\right\}_{j=1}^{N}, N \in \mathbf{N}$, be closed operators on a Hilbert space on $\mathscr{X}$. Suppose that $\cap_{j=1}^{N} \mathscr{D}\left(C_{j}\right)$ is dense in $\mathscr{X}$ and for $j \neq l,\left(C_{j} \Psi, C_{l} \Psi\right)+\left(C_{l} \Psi, C_{j} \Psi\right)=0, \Psi \in \cap_{j=1}^{N} \mathscr{D}\left(C_{j}\right)$. Then $C=\sum_{j=1}^{N} C_{j}$ is closed.

Proof. We see that $(C \Psi, C \Psi)=\sum_{j=1}^{N}\left\|C_{j} \Psi\right\|^{2} \geq(1 / N)$ $\left(\sum_{j=1}^{N}\left\|C_{j} \Psi\right\|\right)^{2}$. Then, $\sum_{j=1}^{N}\left\|C_{j} \Psi\right\| \leq \sqrt{N}\|C \Psi\|$. Then from a closedness criterion (e.g., [1, Theorem B1], [12, Proposition 1]), $C$ is closed. 
From an argument of quadratic forms, there exists a selfadjoint operator $L$ on $\mathscr{H}$ such that $L \geq 1, \mathscr{D}(\sqrt{L})=\mathscr{D}_{0}$ and for all $\Phi, \Psi \in \mathscr{D}(\sqrt{L})$,

$$
(\sqrt{L} \Phi, \sqrt{L} \Psi)=\sum_{j=1}^{N}\left(P_{j} \Phi, P_{j} \Psi\right)+(\Phi, \Psi) .
$$

Lemma 6. Assume (S.1). Then, for all $\Phi, \Psi \in \mathscr{D}(\sqrt{L})$,

$$
\left[\sqrt{L}, P_{j}\right]^{0}(\Phi, \Psi)=0, \quad j=1, \ldots, N .
$$

Proof. Since $L$ is positive and self-adjoint, it follows that $\sqrt{L} \Xi=\int_{0}^{\infty}(1 / \sqrt{\lambda})(L+\lambda)^{-1} L \Xi, \Xi \in \mathscr{D}(L)$. Then, for all $\Phi, \Psi \in \mathscr{D}(L)$,

$$
\begin{aligned}
& {\left[\begin{array}{l}
L \\
\hline
\end{array} P_{j}\right]^{0}(\Phi, \Psi)} \\
& =\int_{0}^{\infty} \frac{1}{\sqrt{\lambda}}\left[(L+\lambda)^{-1} L, P_{j}\right]^{0}(\Phi, \Psi) d \lambda \\
& =\int_{0}^{\infty} \sqrt{\lambda}\left[P_{j}, L\right]^{0}\left((L+\lambda)^{-1} \Phi,(L+\lambda)^{-1} \Psi\right) d \lambda \\
& =\sum_{l=1}^{N} \int_{0}^{\infty} \sqrt{\lambda} \\
& \quad \times \quad\left\{\left[P_{j}, P_{l}\right]^{0}\left((L+\lambda)^{-1} \Phi, P_{l}(L+\lambda)^{-1} \Psi\right)\right. \\
& \left.\quad+\left[P_{j}, P_{l}\right]^{0}\left(P_{l}(L+\lambda)^{-1} \Phi,(L+\lambda)^{-1} \Psi\right)\right\} d \lambda .
\end{aligned}
$$

By (S.1) and (17), we have $\left[\sqrt{L}, P_{j}\right]^{0}(\Phi, \Psi)=0$ for all $\Phi, \Psi \in$ $\mathscr{D}(\sqrt{L})$. Note that $\mathscr{D}(L)$ is a core of $\sqrt{L}$, since $L$ is self-adjoint. In addition, for all $\Psi \in \mathscr{D}(\sqrt{L}),\left\|P_{j} \Psi\right\| \leq\|\sqrt{L} \Psi\|, j=$ $1, \ldots, N$. Hence, it follows that $\left[\sqrt{L}, P_{j}\right]^{0}(\Phi, \Psi)=0$ for all $\Phi, \Psi \in \mathscr{D}(\sqrt{L})$. Thus, the proof is obtained.

Proof of Theorem 3. Since $B \otimes M$ is bounded, it is enough to show that $H=\sum_{j=1}^{N} \Gamma_{j} \otimes P_{j}$ is self-adjoint. Let $X=B \otimes \sqrt{L}$. We show that $H$ and $X$ satisfy (C.1)-(C.3) and (I) in Theorem 2. Since $H$ is symmetric and $X$ is self-adjoint, (C.1) is satisfied. Since $\sigma(B)=\{ \pm 1\}$ and $\sqrt{L} \geq 1$, we see that, for all $\Psi \in \mathscr{D}(H)$,

$$
|(\Psi, X \Psi)|=(\Psi,(I \otimes \sqrt{L}) \Psi) \geq(\Psi, \Psi) .
$$

Then, (C.2) is satisfied. Since $\mathscr{D}_{0}=\mathscr{D}(\sqrt{L})$, it follows that $\mathscr{D}(H)=\mathscr{D}(X)$. Then, by (S.3), we see that, for all $\Psi \in \mathscr{D}(H)$,

$$
\begin{aligned}
\|H \Psi\|^{2} & =\sum_{j=1}^{N}\left(\left(I \otimes P_{j}\right) \Psi,\left(I \otimes P_{j}\right) \Psi\right) \\
& \leq\|(I \otimes \sqrt{L}) \Psi\|^{2}=\|X \Psi\|^{2} .
\end{aligned}
$$

Then, $\|H \Psi\| \leq\|X \Psi\|$ for all $\Psi \in \mathscr{D}(H)$, and hence (C.3) is satisfied. By Lemma 6, it is seen that, for all $\Psi \in \mathscr{D}(H)$,

$$
\begin{aligned}
(H \Psi, X \Psi)+(X \Psi, H \Psi) \\
=\sum_{j=1}^{N}\left(\left(\left(\Gamma_{j} \otimes P_{j}\right) \Psi,(B \otimes \sqrt{L}) \Psi\right)\right. \\
\left.\quad+\left((B \otimes \sqrt{L}) \Psi,\left(\Gamma_{j} \otimes P_{j}\right) \Psi\right)\right) \\
=\sum_{j=1}^{N}\left((I \otimes \sqrt{L}) \Psi,\left(\left\{\Gamma_{j}, B\right\} \otimes P_{j}\right) \Psi\right) .
\end{aligned}
$$

Then by (S.3) and (20), we have $(H \Psi, X \Psi)+(X \Psi, H \Psi)=0$. Then, from (18), it follows that $(H \Psi, X \Psi)+(X \Psi, H \Psi) \leq$ $|(\Psi, X \Psi)|$ for all $\Psi \in \mathscr{D}(H)$. Then (I) is satisfied, and hence $\bar{H}$ is self-adjoint from Theorem 2. In addition, by (S.1) and (S.3), we see that, for $j \neq l$,

$$
\begin{aligned}
& \left(\left(\Gamma_{j} \otimes P_{j}\right) \Psi,\left(\Gamma_{l} \otimes P_{l}\right) \Psi\right)+\left(\left(\Gamma_{l} \otimes P_{l}\right) \Psi,\left(\Gamma_{j} \otimes P_{j}\right) \Psi\right) \\
& \quad=\left(\left(I \otimes P_{l}\right) \Psi,\left(\left\{\Gamma_{j}, \Gamma_{l}\right\} \otimes P_{j}\right) \Psi\right)=0 .
\end{aligned}
$$

Then, from Lemma $5, \bar{H}=H$, and hence the proof is obtained.

\section{Conflict of Interests}

The author declares that there is no conflict of interests regarding the publication of this paper.

\section{Acknowledgments}

It is a pleasure to thank Professor Akito Suzuki and Professor Fumio Hiroshima for their comments. This work is supported by JSPS Grant 24:1671.

\section{References}

[1] A. Arai, Mathematical Principles of Quantum Phenomena, Asakura-shoten, 2005, (Japanese).

[2] M. Reed and B. Simon, Methods of Modern Mathematical Physics, vol. 2, Academic Press, 1975.

[3] J. Glimm and A. Jaffe, "The $\lambda\left(\varphi^{4}\right)_{2}$ quantum field theory without cutoffs. IV: perturbations of the Hamiltonian," Journal of Mathematical Physics, vol. 13, no. 10, pp. 1568-1584, 1972.

[4] E. Nelson, "Time-ordered operator products of sharp-time quadratic forms," Journal of Functional Analysis, vol. 11, no. 2, pp. 211-219, 1972.

[5] W. Faris and R. Lavine, "Commutators and self-adjointness of Hamiltonian operators," Communications in Mathematical Physics, vol. 35, no. 1, pp. 39-48, 1974.

[6] J. Fröhlich, "Application of commutator theorems to the integration of representations of Lie algebras and commutation relations," Communications in Mathematical Physics, vol. 54, no. 2, pp. 135-150, 1977.

[7] B. Thaller, The Dirac Equation, Springer, 1992. 
[8] A. Arai, "Characterization of anticommutativity of self-adjoint operators in connection with clifford algebra and applications," Integral Equations and Operator Theory, vol. 17, no. 4, pp. 451463, 1993.

[9] A. Arai, "Heisenberg operators, invariant domains and Heisenberg equations of motion," Reviews in Mathematical Physics, vol. 19, no. 10, pp. 1045-1069, 2007.

[10] S. Pedersen, "Anticommuting self-adjoint operators," Journal of Functional Analysis, vol. 89, no. 2, pp. 428-443, 1990.

[11] F.-H. Vasilescu, "Anticommuting self-adjoint operators," Revue Roumaine de Mathématiques Pures et Appliquées, vol. 28, no. 1, pp. 76-91, 1983.

[12] J. Glimm and A. Jaffe, "Singular perturbations of selfadjoint operators," Communications on Pure and Applied Mathematics, vol. 22, pp. 401-414, 1969. 


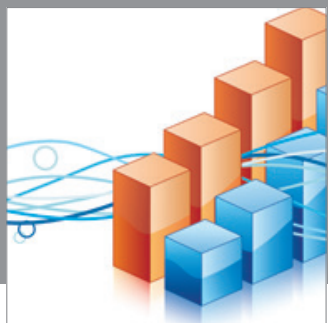

Advances in

Operations Research

mansans

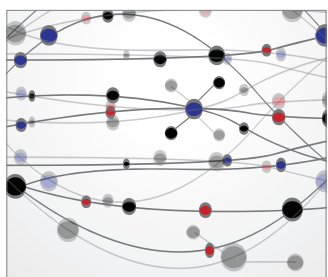

The Scientific World Journal
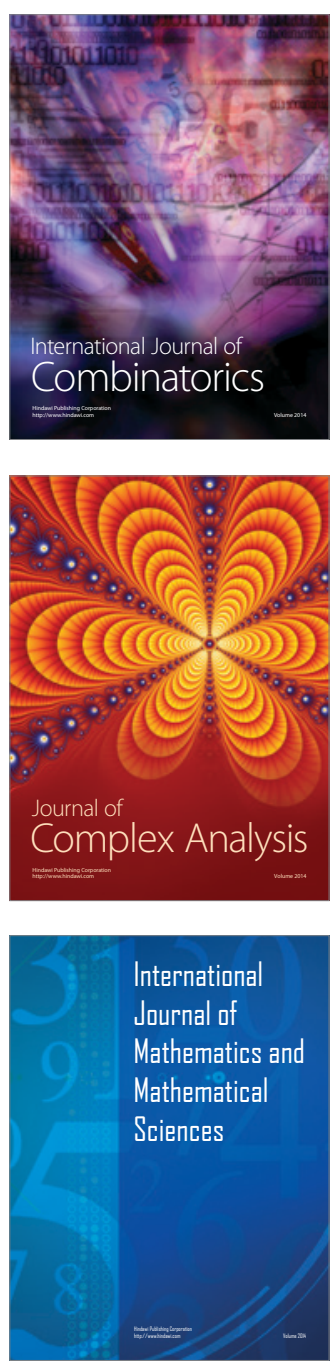
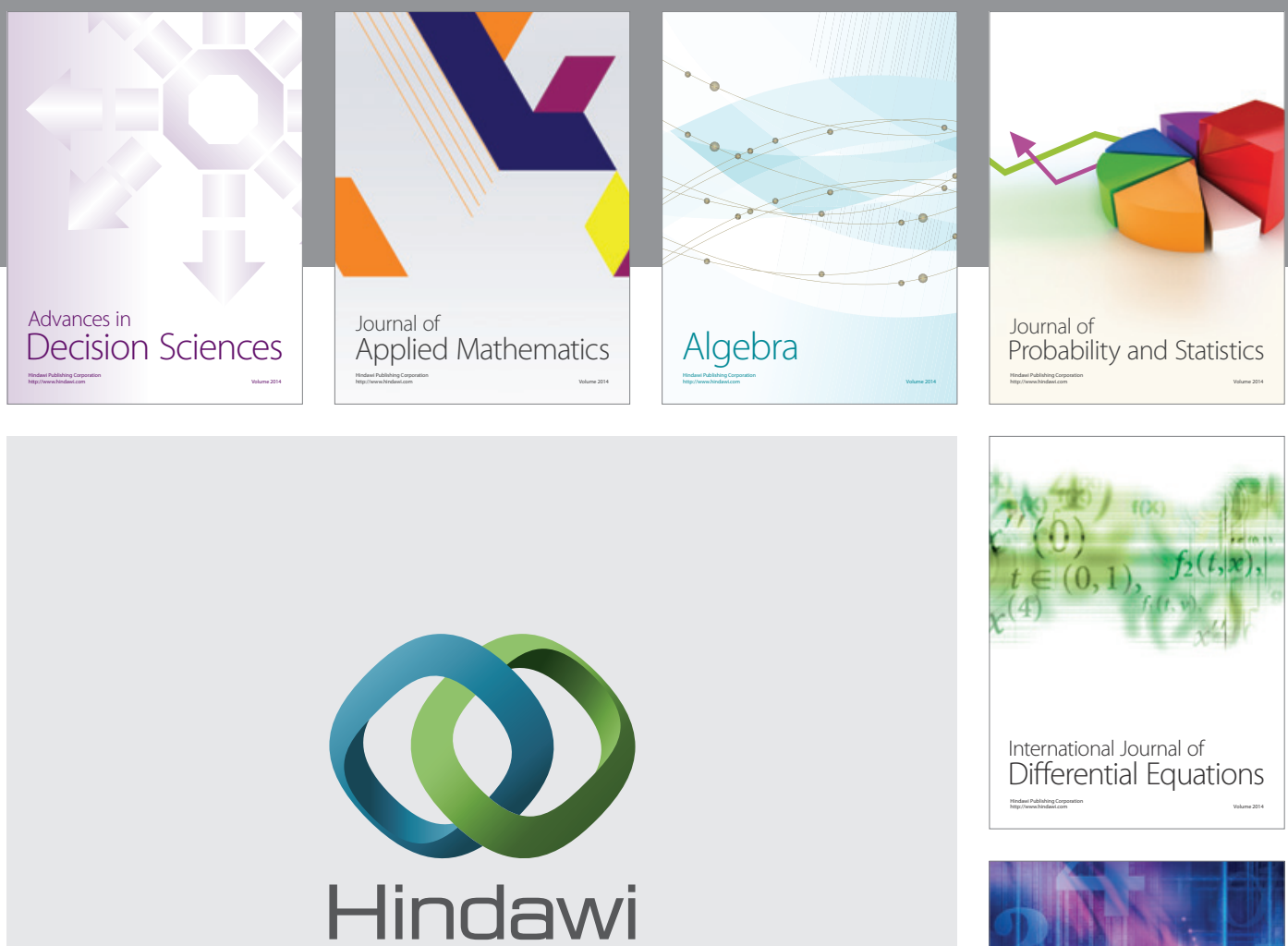

Submit your manuscripts at http://www.hindawi.com
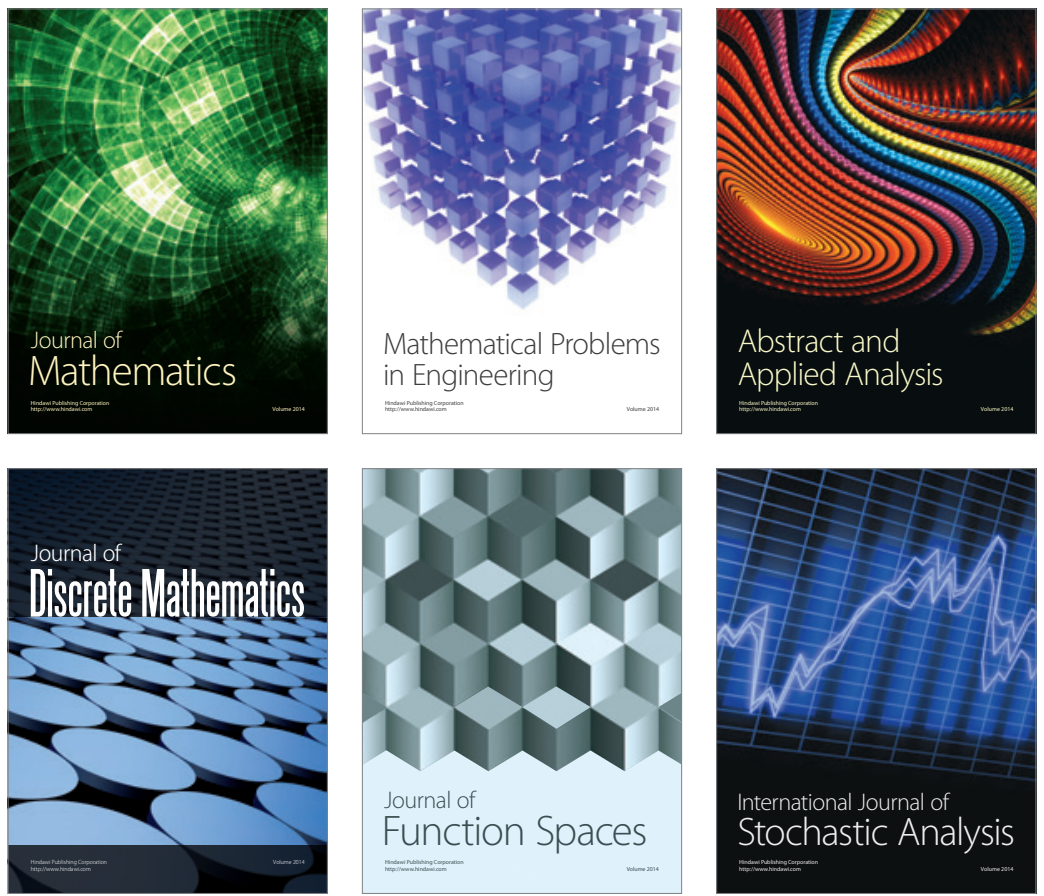

Journal of

Function Spaces

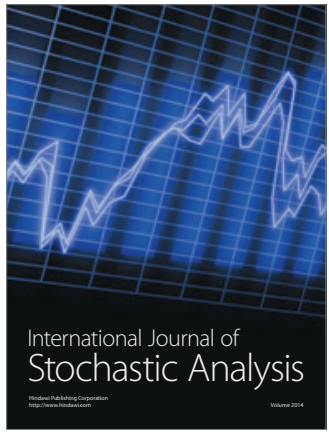

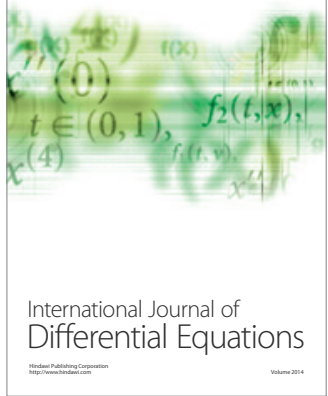
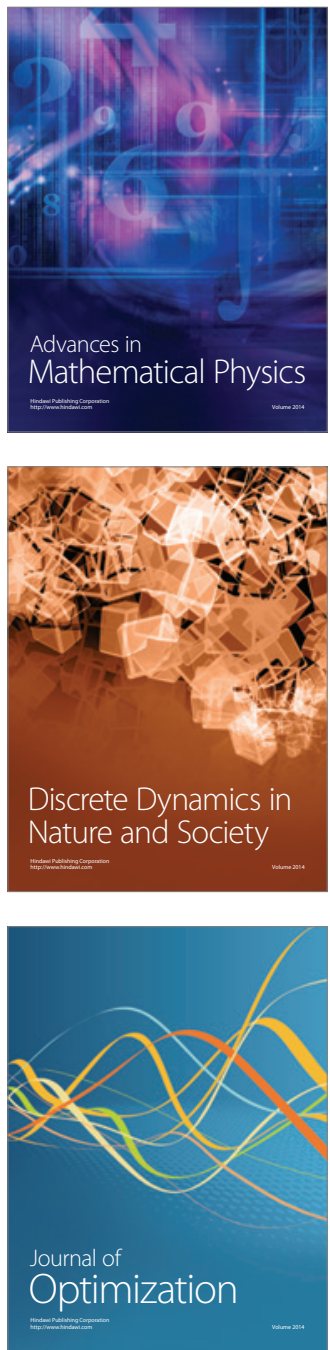